\title{
Avaliação da capacidade laborativa em periciandos portadores de fibromialgia
}

\author{
Evaluation of working capacity in people with fibromyalgia
}

\author{
Clarissa Mari de Medeiros', Talita Zerbini², Raquel Barbosa Cintra², \\ Victor Alexandre Percínio Gianvevecchio ${ }^{3}$, Daniel Romero Muñoz ${ }^{4}$
}

\begin{abstract}
Medeiros CM, Zerbini T, Cintra RB, Gianvevecchio VAP, Muñoz DR. Avaliação da capacidade laborativa em periciandos portadores de fibromialgia. Saúde, Ética \& Justiça. 2012;17(2):50-6.
\end{abstract}

\begin{abstract}
RESUMO: Solicitações de benefícios são frequentemente realizadas em decorrência dos sintomas da fibromialgia, que é a síndrome de dor crônica difusa mais prevalente na população geral, reconhecida pela Organização Mundial da Saúde (OMS) desde 1992. O perito judicial defronta-se, rotineiramente, com dois quesitos básicos: - 1) A fibromialgia é uma doença que acarreta incapacidade laborativa? 2) Tal doença tem nexo causal com alguma atividade laborativa? O presente trabalho tem por objetivo verificar o conceito atual da fibromialgia, bem como determinar critérios médico-legais que permitam a avaliação da capacidade laborativa e do nexo causal com as atividades laborativas exercidas por periciandos diagnosticados com fibromialgia. Para tanto, foi realizada revisão bibliográfica baseada em publicações recentes nas principais bases de pesquisa (Pubmed, SciELO e Lilacs). A doença está enquadradada no grupo que correspondeu a um total de 7.756 benefícios de auxílio-doença e 970 de auxílio-doença acidentário concedidos no ano de 2011, e 1.927 benefícios de auxílio doença e 242 benefícios de auxílio-doença acidentário até março de 2012, pela Previdência Social. De acordo com dados da literatura, a prevalência estimada da doença é de 0,66 a $4 \%$ da população, atingindo preferencialmente o sexo feminino. Os critérios diagnósticos atuais não contemplam mais os tender points, definidos pelo Colégio Americano de Reumatologia (ACR) em 1990, e englobam os sintomas não relacionados ao aparelho locomotor, além da dor musculo-esquelética, que avaliam a sua intensidade. $A$ relação da doença com a atividade ocupacional é alvo de discussões, uma vez que condições como doenças osteomusculares relacionadas ao trabalho (DORT), doenças somatoformes e distúrbios psiquiátricos costumam ser diagnósticos diferenciais difíceis de serem distinguidos. Exames propedêuticos e complementares não elucidam completamente o diagnóstico. Diante desses elementos, os autores deste trabalho discorrem sobre as repercussões funcionais da doença que possam justificar concessão de benefícios como aposentadoria por invalidez ou auxílio doença e discutem os critérios médico-periciais para avaliação do nexo causal entre a fibromialgia e a atividade laborativa exercida por periciandos portadores da doença. Pôde-se concluir que a fibromialgia pode ser incapacitante devido à intensidade da dor e demanda física da função do periciando. Dessa forma, para estabelecer nexo ocupacional, há necessidade de avaliação da atividade laborativa exercida e do ambiente do trabalho, além da exclusão das demais hipóteses diagnósticas. A avaliação da ocorrência de quadros similares em pessoas que exerçam a mesma função que o autor também é importante, uma vez que tal fato gera maior credibilidade à avaliação do nexo.
\end{abstract}

DESCRITORES: Fibromialgia; Prova pericial/legislação \& jurisprudência; Previdência social/legislação \& jurisprudência; Medicina legal/legislação \& jurisprudência.

\footnotetext{
${ }^{1}$ Médica Residente de Medicina do Trabalho da Faculdade de Medicina da Universidade de São Paulo.

${ }^{2}$ Médica preceptora da Residência de Medicina do Trabalho e de Medicina Legal da Faculdade de Medicina da Universidade de São Paulo.

${ }^{3}$ Médico Perito da Vara de Acidentes do Trabalho da Comarca de São Paulo.

${ }^{4}$ Professor Titular do Departamento de Medicina Legal, Ética Médica e Medicina Social e do Trabalho da Faculdade de Medicina da Universidade de São Paulo.
}

Endereço para correspondência: Clarissa Mari de Medeiros. Avenida Doutor Arnaldo, 455, Cerqueira César - São Paulo, SP. CEP: 012903-000. E-mail: clarissamari@ hotmail.com. 


\section{INTRODUÇÃO}

A fibromialgia é uma entidade sindrômica de caráter crônico, não inflamatória, intermitente, de etiologia controversa, que se manifesta principalmente no sistema músculo-esquelético, podendo se associar a distúrbios do sono, fadiga crônica, cefaléia, rigidez matinal, ansiedade e depressão. O sexo feminino é o mais acometido, tendo relação de 6 mulheres para 1 homem. Sua prevalência na população geral varia de 0,66 a $4 \%{ }^{1}$.

Segundo Ferreira et al. $^{2}$, a complexa sintomatologia da fibromialgia envolve principalmente três áreas: sistema músculoesquelético, neuroendócrino e psicológico. Os critérios do Colégio Americano de Reumatologia (ACR) foram elaborados em 1990, e contemplam para classificação de fibromialgia: dor difusa presente no esqueleto axial e em ambos os hemicorpos (acima e abaixo da cintura), dor em 11 ou mais dos 18 tender points e dor crônica por mais de três meses, de acordo com o Projeto Diretriz Sociedade Brasileira de Reumatologia, em 2004 Os tender points são locais mais sensíveis à dor, localizados conforme figura abaixo:

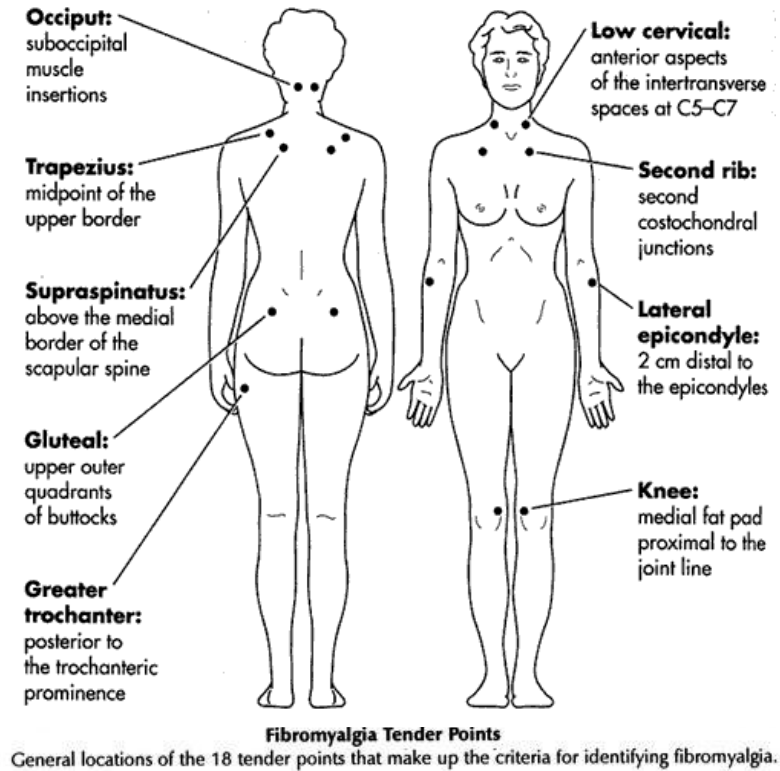

Fonte: http://destinedfor150.blogspot.com/2010/07/five-bags-offlour.html

No entanto, apesar de ser a opção de método diagnóstico de reumatologistas e fisiatras, esses critérios não são usados por todos os especialistas, uma vez que há controvérsias na definição da doença e na distinção da mesma em relação a outras síndromes ${ }^{4}$. Segundo Wolfe apud Aragon ${ }^{5}$, a palpação dolorosa dos tender points não traduz especificidade da síndrome fibromiálgica, sendo o diagnóstico variável de acordo com a pressão exercida nos pontos.

De acordo com Katz et al. ${ }^{6}$, os critérios contemplados pelo ACR não atingem a essência da fibromialgia. Por se tratar de uma síndrome complexa e de manifestação clínica variável, existem autores que até mesmo questionam a existência da fibromialgia como doença $a^{5,7,8}$.

D'Arcy ${ }^{4}$ critica os critérios do ACR, defendendo um novo método que não requeira exame físico ou avaliação dos tender points, justificando que tal exame é frequentemente realizado de forma incorreta. Outros artigos já concluem que a termografia infravermelha como exame complementar pode servir como critério diagnóstico mais fidedigno para o esclarecimento da fibromialgia8 .

Giesecke e Clauw apud Araujo ${ }^{9}$ reconhecem, ainda, que os indivíduos que preenchem os critérios do ACR de classificação para fibromialgia não compõem um grupo homogêneo. Defendem a hipótese de existir distintos grupos entre os fibromiálgicos, classificados pelos quesitos sensibilidade/dor, humor e cognição.

Os considerados "novos" critérios para o diagnóstico de fibromialgia não contemplam os pontos dolorosos (tender points), criticados pelos autores acima descritos. De acordo com essa nova proposta, apresentada em forma de questionário, o paciente deverá preencher três critérios (dor, duração dos sintomas e exclusão de demais diagnósticos com semelhantes sintomas). O primeiro critério é relacionado a uma escala de locais de dor pelo corpo, na qual o paciente deverá ter uma pontuação maior que sete (valor máximo 19), e de um questionário de gravidade de sintomas que incluem fadiga, sono não reparador, sintomas cognitivos e sintomas somáticos com pontuação maior que cinco (valor máximo 12). Outra pontuação válida é a escala de dor de 3 a 6 (valor máximo 19) e a escala de sintomas maior ou igual a nove (valor máximo de 12). Os sintomas devem estar presentes por pelo menos há três meses e o paciente não deve ter um outro distúrbio que possa justificar o aparecimento dos sintomas ${ }^{10}$.

As dificuldades diagnósticas da fibromialgia encontradas na prática médica assistencial também são enfrentadas pelos médicos peritos em diversas esferas. Segundo o Ministério da Previdência nos períodos de 2004 a $2012^{11}$, a fibromialgia esteve entre as três principais causas de concessão de benefícios solicitados no Instituto Nacional do Seguro Social (INSS). Entretanto, sabe-se que a caracterização pericial da doença e a avaliação da 
capacidade laborativa do periciando são complexas.

\section{OBJETIVOS}

\section{Objetivo primário}

Discutir o conceito atual da fibromialgia e os critérios médico-legais definidos que possam permitir estabelecer se a fibromialgia pode gerar redução da capacidade laborativa.

\section{Objetivo secundário}

Avaliar possibilidade de estabelecer nexo causal com as atividades ocupacionais exercidas pelos periciandos diagnosticados com fibromialgia.

\section{MÉTODOS}

O presente trabalho foi elaborado a partir de revisão de literatura nas bases de dados Pubmed, SciELO, CAPES e Lilacs.

Foram selecionados 36 artigos a partir da leitura de seus resumos, além do estudo do Projeto Diretrizes da Sociedade Brasileira de Reumatologia e de dados presentes no site do Ministério da Previdência Social referentes ao período de 2004 a 2012. As palavras-chave utilizadas para a pesquisa foram "fibromialgia", "jurisprudência" e "previdência social', cujos correspondentes em inglês são "fibromyalgia", "jurisprudence" e "social security".

Critérios de inclusão dos artigos basearamse na data - a partir de 2002, tema associado com avaliação pericial, aspectos ocupacionais, origem e prevalência da doença nas línguas inglesa, portuguesa e francesa. Excluídos artigos que detinham no título sugestões ou informações sobre tratamento da doença, e nos demais idiomas.

\section{DISCUSSÃO}

Segundo dados do Ministério da Previdência Social, no ano de 2011 foram registrados 7.756 benefícios de auxílio-doença e 970 de auxíliodoença acidentário associados ao CID-10 M79.7 (fibromialgia). Até março de 2012, já foram registradas 1.927 concessões de benefícios tipo auxílio-doença e 242 auxílio-doença acidentário pelas mesmas causas ${ }^{11}$.

O fato de a fibromialgia não ser considerada doençaporalgunsautoresbaseia-senapossibilidade da mesma apenas fazer parte de um espectro de respostas alteradas a um fator estressor. Desse modo, os peritos médicos poderiam desconsiderála como causa de incapacidade laborativa ${ }^{12}$. A Ordem de Serviço do Instituto Nacional do Seguro Social (INSS) publicada em 1998 apresenta visões contraditórias a respeito do conceito da doença: "pode-se concluir que a fibromialgia é uma doença complexa que pode ter ou não um componente ocupacional importante". Entretanto, na segunda seção do mesmo documento, a fibromialgia foi classificada como "patologia não ocupacional"22.

Dados da década de 90 já apontavam a fibromialgia como patologia importante na solicitação e na concessão de benefícios sob alegação de incapacidade funcional e ocupacional: $15 \%$ dos pacientes estudados com este diagnóstico receberam benefícios nos Estados Unidos; 24\% dos pacientes suecos receberam algum tipo de pensão e $50 \%$ dos pacientes ingleses pararam de trabalhar por conta das repercussões funcionais do diagnóstico ${ }^{13}$. O mesmo trabalho mostra que, no Canadá, a fibromialgia já foi responsável por 9\% de todos os benefícios denominados "disability payments". Estudo de Wolfe e Potter ${ }^{14}$ encontrou $25,3 \%$ dos pacientes recebendo algum benefício, entre os chamados "disability payments and requirements". De acordo com Ferreira et $\mathrm{al}^{2}$, isso ocorre pelas repercussões geradas pela dor e intolerância ao exercício físico que reprimem a habilidade para o trabalho e execução das atividades funcionais.

Os números citados revelam a importância do tema e a urgência em se pesquisar e determinar aspectos-chave da fibromialgia, tais como diagnóstico preciso, etiopatogenia e correlação com o trabalho exercido. White et al. ${ }^{13}$ discorre sobre fatores que contribuem para disfunção ou incapacidade provocadas pela doença, tais como dor crônica e desconforto autopercebido, prejuízo da memória e concentração e associação freqüente com migrânea e demais síndromes (bexiga irritável, entre outros). Os aspectos controversos descritos nesse mesmo trabalho aparecem na avaliação da incapacidade supostamente promovida pela fibromialgia, pois levam em consideração a falta de aceitação da entidade como doença, a postura do médico-perito, a presença de anormalidades psicológicas, a discrepância entre relato do paciente/ periciando e os exames complementares, além do papel do trabalho na etiogênese ou agravamento da doença.

Dados da Organização Mundial da Saúde apud Martinez ${ }^{12}$ demonstram que a dor crônica acomete um número expressivo de pessoas, sendo uma das principais causas da incapacitação física para o trabalho. Estudos mostram que 10\% da 
população mundial sofrem de dor crônica difusa, dentre os quais 3 a $5 \%$ correspondem a casos de fibromialgia ${ }^{15}$, descrita como a doença de dor crônica mais comum atualmente ${ }^{16}$.

Estudos relatam correlação entre a contagem dos pontos e a intensidade da dor e capacidade funcional ${ }^{6,17}$. O método de diagnóstico recomendado pelo ACR é alvo de crítica em alguns estudos, que revelam que a palpação dolorosa não é efetivamente realizada na prática clínica durante exame físico, mostrando que $80 \%$ dos casos de fibromialgia são diagnosticados independentemente da recomendação da $\mathrm{ACR}^{16}$. Atualmente, critérios para 0 diagnóstico de fibromialgia não contemplam os pontos dolorosos (tender points). São apresentados na forma de questionário, em que o paciente deve preencher três critérios (dor, duração dos sintomas e exclusão de demais diagnósticos com semelhantes sintomas). O primeiro critério é relacionado a uma escala de locais de dor pelo corpo, na qual o paciente deverá ter uma pontuação maior que sete (valor máximo 19), e de um questionário de gravidade de sintomas que incluem fadiga, sono não reparador, sintomas cognitivos e sintomas somáticos uma pontuação maior que cinco (valor máximo 12). Outra pontuação válida é a escala de dor de 3 a 6 (valor máximo 19) e a escala de sintomas maior ou igual a nove (valor máximo de 12). Os sintomas devem estar presentes por pelo menos três meses e o paciente não deve ter um outro distúrbio que possa justificar o aparecimento dos sintomas ${ }^{10}$.

A Sociedade Americana de Dor recomenda, ainda, que se obtenham exames complementares, tais como hemograma completo, velocidade de hemossedimentação, enzimas musculares, função hepática, função tireoidiana em pacientes que não apresentarem, pelos critérios da ACR, o mínimo de 11 pontos, mas que têm forte sugestão do diagnóstico de fibromialgia.

Como opções de avaliação sintomatológica da ampla variedade de apresentações clínicas que a fibromialgia detém, apresentam-se questionários e escalas, úteis no estabelecimento do diagnóstico. $\mathrm{Em}$ relação à dor, queixa mais frequente $\mathrm{e}$ característica da doença, há confiabilidade na utilização de escalas analógicas, sendo a mais frequentemente citada a escala analógica visual (VAS), composta por uma linha de $10 \mathrm{~cm}$, sendo uma das extremidades representada pela ausência de dor, e a outra extremidade, a maior intensidade possível do sintoma. É solicitado ao paciente que escolha o valor que melhor corresponde à queixa referida $^{3,6,16}$.

A avaliação da qualidade de vida ou impacto da doença nas atividades cotidianas pode ser feita pelo Fibromyalgia Impact Questionnaire (FIQ), composto de questões relacionadas à influência da doença nas atividades do dia-a-dia, nas atividades profissionais e questões relacionadas à intensidade dos principais sintomas, considerado como de boa confiabilidade ${ }^{3}$.

O questionário de qualidade de vida conhecido como Medical Outcome Survey 36 Itens Short Form Study - SF36 também pode ser válido, porém a subjetividade que o acompanha não garante a veracidade da dor referida $e$, consequentemente, da incapacidade que pode gerar $^{2,8}$. No entanto, estudos apontam que analisar os possíveis sintomas psicológicos secundários à fibromialgia é fundamental para saber se a dor referida pelos pacientes é real ${ }^{18}$.

O nível de conhecimento a respeito da patogênese da fibromialgia apresentou progresso significativo nas últimas décadas. De acordo com o Projeto Diretrizes ${ }^{3}, 30$ a $50 \%$ dos pacientes com fibromialgia apresentam depressão associada ao quadro. Alguns outros fatores já foram considerados na fisiopatologia da fibromialgia, tais como agentes infecciosos (HIV, Hepatite B e $\mathrm{C}$ ), doenças reumatológicas (artrite reumatóide, Sjögren), desbalanços nos níveis de alguns neurotransmissores, citocinas e hormônios. Entretanto, os estudos que os mencionam não foram conclusivos a esse respeito ${ }^{18,19}$.

Pelo fato de a fibromialgia não apresentar definição precisa, muitas outras condições similares podem surgir como diagnósticos diferenciais, tais como síndrome da dor miofascial, reumatismo extra-articular, polimialgia reumática e artrite de células gigantes, polimiosites e dermatopolimiosites, hipotireoidismo, hipertireoidismo, hiperparatireoidismo, insuficiência adrenal, neoplasias, miopatias por álcool e efeitos colaterais de medicamentos. Além disso, existe a possibilidade de "overlappings", que são duas ou mais condições reumatológicas que coexistem em um mesmo indivíduo, tornando o diagnóstico ainda mais difícil ${ }^{3}$.

Além dos fatores citados acima, a avaliação da saúde mental dos periciandos portadores de fibromialgia é fundamental para a caracterização da síndrome, uma vez que sintomas psíquicos estão associados à doença em 20 a $50 \%$ dos $\operatorname{casos}^{14}$. Cosci et al. ${ }^{20}$ inicia seu estudo caracterizando a fibromialgia como síndrome somática relacionada ao estresse, com fatores psicológicos que podem interferir, de maneira ainda não muito clara, na evolução da doença e na capacidade laboral dos periciandos. 
Confusões diagnósticas são verificadas em outros estudos, que garantem que apenas $34 \%$ dos pacientes considerados portadores da doença efetivamente a possuem, sendo os demais prováveis portadores de espondiloartrites soronegativas, tendinites, lúpus eritematoso sistêmico (LES), artrite reumatoide $(A R)$ e osteoartrite ${ }^{3}$.

Apesar de existir crítica a respeito da hipervalorização de exames complementares no diagnóstico de algumas doenças ${ }^{12}$, a utilização dos mesmos pode ser útil em perícias médicas para casos complexos e controversos como os de fibromialgia, uma vez que o exame físico detém valor subjetivo e pode ser simulado pelo periciando solicitador de benefício. Exames de eletroneuromiografia (ENMG), ultrassonografia (US), tomografia (TC) e ressonância magnética (RNM) são frequentemente solicitados nos casos menos característicos e quando há necessidade de descartar outras entidades nosológicas. As indicações desses exames complementares não são absolutas, mas esses ainda são constantemente requeridos diante das incertezas dos métodos diagnósticos, falta de padronização e das queixas vagas provenientes dos periciandos ${ }^{21}$.

Brioschi et al. ${ }^{8}$ pôde estabelecer correlação entre a clínica dos pacientes, seguindo os critérios da ACR, com o exame de termografia infravermelha - método diagnóstico que, por meio de sensor acoplado a um sistema computadorizado, mensura a distância da radiação infravermelha emitida pela superfície cutânea, com sensibilidade de $0,07^{\circ} \mathrm{C}$, o que representa relação direta com a microcirculação cutânea sob controle do sistema neurovegetativo cutâneo. Outros estudos consideram que 0 método oferece um critério objetivo no diagnóstico complementar da fibromialgia, uma vez que esta apresenta um padrão hiper-radiante difuso correspondente às áreas musculares dolorosas, o que difere da hiper-radiação localizada encontrada na síndrome da dor miofascial ${ }^{7}$.

A avaliação da capacidade laboral, considerando a presença da dor fibromiálgica, é realizada de acordo com a função exercida pelo periciando. Estudos relatam que a clínica mostra cada vez mais pacientes fibromiálgicos com alguma queixa de sobrecarga ocupacional, mas poucos trabalhos são encontrados na literatura que apontem para uma possível evolução da doença que promova sintomas apenas locais, típicos de lesão por esforço repetitivo (LER) e doença osteomuscular relacionada ao trabalho (DORT) ${ }^{22}$. Em muitas situações, o nexo causal com o trabalho foi descartado no momento em que foi realizado o diagnóstico de fibromialgia, uma vez que o nexo somente era estabelecido quando diagnosticado DORT. Este diagnóstico diferencial foi mencionado na discussão do caso em estudo, uma vez que documentos apresentados pela pericianda sugeriam positividade para tenossinovite de De Quervain em um dos membros superiores.

Rezende ${ }^{23}$ acredita que o fato de existir uma doença de base facilitadora de dor, tal como a fibromialgia, pode iniciar ou perpetuar um quadro inicialmente ocupacional, devendo assim ser aberta a Comunicação de Acidente de Trabalho (CAT), tal qual um acidente de trabalho. Da mesma forma, o fato de o trabalho piorar uma dor preexistente também deve ser considerado. Tal raciocínio pode ser estendido para afastamento do trabalho ou solicitação de benefícios. Martinez ${ }^{12}$ afirma que, nos casos em que um ambiente inadequado se associa à insatisfação pessoal com a atividade, pode ser desencadeada a fibromialgia.

Wolfe e Potter ${ }^{14}$, em contrapartida, relatam que, embora haja tendência crescente em aceitar a fibromialgia como doença, a associação dos seus sintomas com incapacidade laborativa não pode ser feita. Para White et al. $^{13}$ o trabalho não pode ser categoricamente considerado como causa da fibromialgia. Entretanto, também menciona que sejam feitas mudanças no ambiente do trabalho, readaptação profissional e redução da carga horária para funcionários com quadro sugestivo da síndrome de dor crônica. Já Reisine et al. ${ }^{24}$ e Rivera et al. ${ }^{25}$ concluem que os pacientes portadores de fibromialgia que continuam trabalhando apresentam melhor resposta clínica ao tratamento quando comparado àqueles que não trabalham. Isso sugere que o trabalho é fator positivo no prognóstico e atua de forma benéfica na diminuição da intensidade dos sintomas provocados pela fibromialgia ${ }^{26}$. Helfenstein et $\mathrm{al}^{27}$ relata haver evidências negativas para o paciente com essa doença quanto a litigância e aos afastamentos prolongados.

Wolfe e Potter ${ }^{14}$ mostram que há reconhecimento da natureza subjetiva da dor por parte dos juízes, além de reconhecer as limitações do exame físico pericial para documentar a fibromialgia e a sua gravidade. Estudos que propuseram a análise da percepção judicial nos casos litigiosos envolvendo fibromialgia mostram diversas opiniões. Page et al. ${ }^{28}$ mostraram que $91 \%$ das causas foram acatadas pelo juiz com base na credibilidade do periciando, na maneira de apresentação da solicitação dos benefícios, na investigação complementar da queixa e no interrogatório de testemunhas. A conclusão mostra, novamente, que devido à complexidade 
envolvendo a doença, o sistema legal e securitário continuarão a apresentar dificuldades nas questões que envolvam a fibromialgia.

\section{CONCLUSÃO}

A fibromialgia, reconhecida pela Organização Mundial da Saúde desde 1992, apresenta prevalência significativa tanto na medicina assistencial e preventiva, como na normativa. O método diagnóstico, a avaliação da incapacidade funcional e o estabelecimento do nexo causal com o trabalho são questões controversas em âmbito mundial. As mesmas dificuldades são enfrentadas tanto pelos clínicos, como pelos peritos.

De acordo com o exposto no presente trabalho, os exames complementares podem auxiliar a avaliação e o diagnóstico médico-legal no momento da perícia. A termografia infravermelha tem trazido mais objetividade ao diagnóstico da doença e, somada aos exames de imagem convencionais, fornece mais informações ao perito para que este possa correlacionar o quadro clínico com a capacidade funcional e avaliar a presença do nexo causal com o trabalho. Além disso, outras condições patológicas de mesma sintomatologia podem ser excluídas por esses exames, conferindo maior veracidade ao diagnóstico de fibromialgia.

A dor difusa relatada, sintoma essencial na síndrome fibromiálgica, é parâmetro subjetivo, que tende a ser mais bem compreendido e interpretado com o resultado da avaliação psíquica e o uso dos questionários citados no presente trabalho, sendo este último restrito, até o momento, a estudos $\mathrm{e}$ pesquisas relacionados ao tema.

A fibromialgia pode ser incapacitante devido à intensidade da dor e demanda física da função do periciando. Dessa forma, para estabelecer nexo ocupacional, há necessidade de avaliação da atividade laborativa exercida e do ambiente do trabalho, além da exclusão das demais hipóteses diagnósticas.

Medeiros CM, Zerbini T, Cintra RB, Gianvevecchio VAP, Muñoz DR. Evaluation of working capacity in people with fibromyalgia. Saúde, Ética \& Justiça. 2012;17(2):50-6.

\begin{abstract}
Social benefits are often requested due to symptoms of fibromyalgia, the most prevalent chronic widespread pain syndrome in the general population, recognized by the World Health Organization (WHO) since 1992. The Court expert is faced routinely with two basic questions: - 1) Is Fibromyalgia a disease that causes labor incapacity? 2) Does this disease have a causal nexus with some labor activity? This study aims to verify the current concept of fibromyalgia as well as determining the criteria that allow medical-legal evaluation of labor incapacity and the causal nexus with the occupational activities performed by people with fibromyalgia. For doing so, literature review was performed based on recent publications in major research bases (Pubmed, Scielo and Lilacs). Fibromyalgia is inserted in the group that corresponded to 7.756 sickness benefits and 970 benefits due to accidents granted in 2010, and 1.927 sickness benefits and 242 benefits due to accidents until March of 2012, by Social Security. According to literature data the estimated prevalence of the disease is 0.66 to $4 \%$ of the population, reaching preferably females. The current diagnostic criteria do not include anymore the tender points, defined by the American College of Rheumatology (ACR) in 1990, and comprehend the symptoms unrelated to the limbs, besides the muscular-skeletal pain, which evaluate their intensity. The relation between this disease and the occupational activity is object of discussion, since conditions such as work-related musculoskeletal disorders (MSDs), somatoform disorders and psychiatric disorders are differential diagnoses that use to be difficult to be distinguished. Propaedeutic and complementary exams do not completely elucidate the diagnosis. Given these elements, the authors of this paper discourse about the functional consequences of the disease that may justify granting benefits like disability retirement or sickness assistance and discuss the medical-expert criteria for assessment of the causal nexus between fibromyalgia and labor activity exercised by people with fibromyalgia. The conclusion of this article is that fibromyalgia can be disabling in consequence of the intensity of the pain and occupational physics demands. Thus, it's necessary to evaluate labor activity and work environment in order to establish a nexus between work and disease, besides excluding other diagnoses hypotheses. The evaluation of the occurrence of similar symptoms in people who works at the same labor conditions that the worker with fibromyalgia is also important, since this information guarantees more credibility to the nexus evaluation.
\end{abstract}

KEYWORDS: Fibromyalgia; Expert testimony/legislation \& jurisprudenc; Social security/legislation \& jurisprudence; Forensic medicine/legislation \& jurisprudence. 


\section{REFERÊNCIAS}

1. Cavalcante A, Sauer J, Chalot S, Assumpção A, Lage $L$, Matsutani L, et al. A prevalência de fibromialgia: uma revisão de literatura. Rev Bras Reumatol. 2006;46(1):40-8.

2. Ferreira EAG, Marques AP, Matsutani LA, Vasconcellos EG, Mendonça LLF. Avaliação da dor e estresse em pacientes com fibromialgia. Rev Bras Reumatol. 2002;42(2):104-10.

3. Sociedade Brasileira de Reumatologia. Projeto Diretrizes - Fibromialgia. São Paulo: Universidade Federal de São Paulo; 2004.

4. D'Arcy Y. New thinking about fibromyalgia pain. Nursing. 2011;41(2):63-4.

5. Aragon L. Fibromialgia: perspectivas de um campo problemático. Interface Comum Saude Educ. 2010;14(32):155-69.

6. Katz RS, Wolfe F, Michaud K. Fibromyalgia diagnosis. Arthritis Rheum. 2006;54(1):169-76.

7. Martinez J, Fujisawa E, Carvalho T, Gianini R. Correlação entre a contagem dos pontos dolorosos da fibromialgia com a intensidade dos sintomas e seu impacto na qualidade de vida. Rev Bras Reumatol. 2009;49(1):32-8.

8. Brioschi ML, Yeng LT, Kasiyama HHS, Pastor EMH, Heupa S, Silva FMRM, et al. Documentação médicolegal da síndrome fibromiálgica: índice termográfico. Rev Dor. 2008;9(4):1327-44.

9. Araújo LA. Fibromialgia: construção e realidade na formação dos médicos. Rev Bras Reumatol. 2006;46(1):56-60.

10. Häuser W, Jung E, Erbslöh-Möller B, Gesmann M, Kühn-Becker $\mathrm{H}$, Petermann $\mathrm{F}$, et al. Validation of the fibromyalgia survey questionnaire within a crosssectional survey. Plos One. 2012;7(5):e37504.

11. Brasil. Ministério da Previdência [citado 30 nov. 2012] Disponível em: http://www.mps.gov.br/index.php.

12. Martinez JE. Fibromialgia: o desafio do diagnóstico correto. Rev Bras Reumatol. 2006;46(1):2.

13. White KP, Harth M, Teasell RW. Work disability evaluation and the fibromyalgia syndrome. Semin Arthritis Rheum. 1995;24(6):371-81.

14. Wolfe F, Potter J. Fibromyalgia and work disability is fibromyalgia a disabling disorder? Rheum Dis Clin North Am. 1996;22(2):369-91.

15. Heymann RE. O papel do reumatologista frente à fibromialgia e à dor crônica musculoesquelética. Rev Bras Reumatol. 2006;46(1):1.

16. Perrot S. Fibromyalgia syndrome: a relevant recente constrution of an ancient condition? Curr Opin in Support Palliat Care. 2008;2(2):122-7.

17. Paiva ES. Fibromialgia. Rev Bras Reumatol. 2003;43(2):112-8.

18. Ablin J, Neumman L, Buskila D. Pathogenesis of fibromyalgia - a review. Joint Bone Spine. 2008;75(3):273-9.

19. Katz R, Block J. Fibromyalgia: update on mechanisms and management. J Clin Rheumatol. 2007;13(2):1029.

20. Cosci F, Pennato T, Bernini O, Berrocal C. Psychological Weel-Being, negative affectivity, and functional impairment in fibromyalgia. Psychother Psychosom. 2011;80(4):256-8.

21. Bombana J. Sintomas somáticos inexplicados clinicamente: um campo impreciso entre a psiquiatria e a clínica médica. J Bras Psiquiatr. 2006;55(4)30812.

22. Alvares T, Antunes M. Fibromialgia - interfaces com as LER/DORT e considerações sobre sua etiologia ocupacional. Cien Saude Colet. 2010;15(3):803-12.

23. Rezende MC. A síndrome fibromialgia no dia-a-dia da medicina ocupacional. Rev Bras Reumatol. 2002; 42(5):318-9.

24. Reisine S, Fifield J, Walsh SJ, Feinn R. Do employment and family work affect the health status of women with fibromyalgia? J Rheumatol. 2003;30(9):2045-53.

25. Rivera J, Esteve-Vives J, Vallejo MA, Rejas J. Factors associated with temporary work disability in patients with fibromyalgia. Rheumatol Int. 2010;31(11):14717.

26. Reisine S, Fifield J, Walsh SJ, Forrest D. Employment and health status changes among women with fibromyalgia: a five-year study. Arthritis Rheum. 2008;59(12):1735-41.

27. Helfenstein Junior M, Goldenfum MA, Siena CAF. Fibromialgia: aspectos clínicos ocupacionais. Rev Assoc Med Bras. 2012;58(3):358-65.

28. Page J, Iverson G, Collins P. The impact of judges' perceptions of credibility in fubrolyalgia claims. Int $\mathrm{J}$ Law Psychiatry. 2008;31(1):30-40. 